\title{
Die kommende Pandemie
}

\section{Konturen eines neuen Seuchenregimes}

\author{
MATthiAs LEANZA
}

\section{Einleitung: Mit Schaden Rechnen}

Der Horizont hat sich verdunkelt. Zukunft scheint heute weniger ein offener Möglichkeitsraum zu sein, in dem gleichermaßen Chancen, die es zu ergreifen gilt, wie zu vermeidende Gefahren auf uns warten. ${ }^{1}$ Vielmehr wirkt das, was auf uns zukommen könnte, für viele zutiefst bedrohlich. Ob man an den Klimawandel, den islamistischen Terrorismus oder die anhaltende Instabilität des Bankenund Finanzsektors denkt, in verschiedenen Bereichen des öffentlichen Lebens wird der kollektive Zukunftsbezug von einer Erwartungshaltung bestimmt, die sich mit Craig Calhoun als »emergency imaginary « ${ }^{2}$ bezeichnen lässt. Demnach rechnen wir, ungefähr seit der Jahrtausendwende, in einer Reihe von Themenund Handlungszusammenhängen, die für das gesellschaftliche Zusammenleben von Bedeutung sind, vermehrt mit der Unvermeidbarkeit von Schäden, um uns so zumindest ein Stück weit auf sie vorbereiten zu können.

Auch wenn sich Zukunftsimaginationen auf potenzielle Ereignisse und $\mathrm{Zu}$ stände beziehen, von denen man jetzt noch nicht wissen kann, ob sie jemals Aktualität erlangen werden, bilden sie ein wichtiges Element in der kulturellen Pro-

$1 \mathrm{Zu}$ diesem für die Moderne insgesamt prägenden Zukunftsmodell vgl. Makropoulos, Michael: Modernität und Kontingenz, München: Fink 1997; Hölscher, Lucian: Die Entdeckung der Zukunft, Frankfurt a.M.: Fischer 1999.

2 Calhoun, Craig: »A World of Emergencies: Fear, Intervention, and the Limits of Cosmopolitan Order«, in: Canadian Review of Sociology/Revue canadienne de sociologie 41 (2004), S. 373-395, hier S. 376. Siehe dazu auch Aradau, Claudia/van Munster, Rens: Politics of Catastrophe: Genealogies of the Unknown, London/New York: Routledge 2011; Horn, Eva: Zukunft als Katastrophe, Frankfurt a.M.: Fischer 2014. 
duktion von Wirklichkeit. ${ }^{3}$ Indem wir uns ein Bild vom morgigen Tag machen, orientieren wir unser heutiges Handeln und Entscheiden in zeitlicher Hinsicht. Dass sich das gesellschaftliche Zukunftsbild seit der Jahrtausendwende verfinstert, dürfte einem Zusammenspiel von neu entstandenen Gefahren und geschärfter Gefahrenwahrnehmung geschuldet sein: Der sich bereits abzeichnende Klimawandel trifft auf eine erhöhte Aufmerksamkeit in Wissenschaft, Politik und Massenmedien, Maßnahmen im Bereich der Terrorprävention werden durch eine verschärfte Gefährdungslage legitimiert, die in der Eurozone seit 2009 regelmäBig durchgeführten Bankenstresstests evaluieren die Krisenfestigkeit des europäischen Bankensystems, um im gegebenen Fall durch frühzeitige Interventionen einen drohenden Systemkollaps zu verhindern, der nunmehr im Bereich des Möglichen zu liegen scheint.

Gegenstand dieses Aufsatzes ist die Verdunklung des Zukunftshorizonts und seine Folgen für das Handeln in einem weiteren Bereich, der von großer Bedeutung für das gesellschaftliche Zusammenleben ist: dem globalen Seuchenschutz. Seit der Jahrtausendwende unternimmt die Weltgesundheitsorganisation in $\mathrm{Zu}$ sammenarbeit mit einer großen Zahl lokaler Akteure rege Anstrengungen, die Weltbevölkerung vor hochansteckenden und zumeist neuartigen Viruserkrankungen zu schützen. Auch wenn man hierbei durch ein möglichst frühzeitiges Eingreifen der Ausweitung eines Ansteckungsherds entgegenzuwirken versucht, rechnen die federführenden Akteure zugleich mit einem Versagen der primären Schutzmaßnahmen. Pandemien, so die Überzeugung, ließen sich nicht sicher verhindern. Dennoch könne man im Sinne der preparedness für den Notfall planen, um dem Unvermeidlichen ein Stück weit seine Schwere zu nehmen. Es handelt sich somit um einen Grenzfall von Prävention: Dass künftig Schäden auftreten, wird als (nahezu) sicher erachtet; das jeweilige Schadensausmaß hingegen gilt als kontingent, das heißt durch Handeln beeinflussbar. ${ }^{4}$

3 Vgl. grundsätzlich dazu Uerz, Gereon: ÜberMorgen. Zukunftsvorstellungen als Elemente der gesellschaftlichen Konstruktion der Wirklichkeit, München: Fink 2006 und mit Blick auf Prävention Willer, Stefan: »Sicherheit als Fiktion. Zur kultur- und literaturwissenschaftlichen Analyse von Präventionsregimen«, in: Markus Bernhardt/ Stefan Brakensiek/Benjamin Scheller (Hg.), Ermöglichen und Verhindern. Vom Umgang mit Kontingenz, Frankfurt a.M./New York: Campus 2016, S. 235-255.

4 Prävention kann es nur dort geben, wo sich mögliche Schäden in der Zukunft durch gegenwärtiges Handeln vermeiden, aufschieben oder zumindest in ihren Folgen abmildern lassen; vgl. dazu Bröckling, Ulrich: »Vorbeugen ist besser... Zur Soziologie der Prävention«, in: Behemoth 1(1) (2008), S. 38-48, hier S. 39f.; Leanza, Matthias: »Prävention«, in: Benjamin Bühler/Stefan Willer (Hg.), Futurologien. Ordnungen des Zukunftswissens, München: Wilhelm Fink 2016, S. 155-167 hier S. 156f. 
Die folgenden Ausführungen nähern sich diesem neuen, gegenwärtig im Entstehen begriffenen Seuchenregime, das Institutionen und Techniken, Wissensordnungen und Normen miteinander verknüpft, um die drohende Gefahr abzuwenden, in drei Schritten: Zunächst möchte ich einige grundsätzliche Überlegungen zum Problem des staatlichen Seuchenschutzes voranstellen, wobei mich insbesondere das Verhältnis von Souveränität, Grundrechten und Biopolitik interessiert. Im Anschluss daran sollen in historischer Perspektive die Grenzen nationalstaatlicher Souveränität angesichts globaler Seuchengefahren betrachtet werden - und wie durch Bildung formaler Organisationen auf Weltebene dennoch eine suprastaatliche Koordination im Kampf gegen Pandemien möglich wird. Vor diesem Hintergrund kann schließlich das neue Seuchenregime, dessen Konturen sich seit einigen Jahren deutlicher abzeichnen, hinsichtlich seines doppelten Zukunftsbezugs und seiner komplexen Organisationsform betrachtet werden.

\section{Zum Problem des Seuchenschutzes IM MODERNEN STAAT}

Die Geschichte moderner Staatlichkeit ist mit der des Seuchenschutzes verschränkt. Nach Thomas Hobbes kann der Souverän - sei dieser eine natürliche Person wie in der Monarchie oder aber eine Versammlung wie in aristokratischen und demokratischen Gemeinwesen ${ }^{5}$ - seine Legitimation allein daraus schöpfen, dass er seinen Untertanen Schutz gewährt. Der künstliche Staatskörper, »jener große Leviathan «, ${ }^{6}$ habe die natürlichen Körper der Menschen, die ihn bildeten, vor Schaden zu bewahren, wolle er selbst nicht zerfallen: »Es versteht sich, daß die Verpflichtung der Untertanen gegen den Souverän so lange und nicht länger dauert, wie die Macht dauert, mit deren Hilfe er sie schützen kann. " $^{7}$

Auch wenn Hobbes hierbei vor allem den Schutz vor Diebstahl und Verbrechen, Gewalt und Krieg im Sinn hatte, tauchte am Rande - oder genauer: sehr klein in der Bildmitte - bereits das Problem des gesundheitlichen Schutzes auf. Das wahrscheinlich von Abraham Bosse geschaffene Titelkupfer des Leviathan

5 Dies sind nach Thomas Hobbes die drei Grundformen staatlicher Gemeinweisen: Hobbes, Thomas: Leviathan, hg. v. Hermann Klenner, Hamburg: Meiner 1996 [1651], S. $156-169$.

6 Ebd., S. 5.

7 Ebd., S. 187. 
von 1651, das als Ikone des neuzeitlichen Staates gelten kann, ${ }^{8}$ enthält nämlich ein bemerkenswertes Detail: Auf dem Kirchenvorplatz in der Stadt, über der der Souverän mit Schwert und Krummstab thront, finden sich zwei Seuchenärzte mit Schnabelmasken, welche an die Pest gemahnen, die im frühneuzeitlichen Europa endemisch geworden war. ${ }^{9}$ Das leicht zu übersehende Bildelement verweist sowohl auf die Bedrohung, die von der Seuche für Leib und Leben ausgeht, als auch auf den Versuch, die Gemeinschaft vor ihr zu schützen, nicht zuletzt mithilfe von Isolations- und Quarantänemaßnahmen, wie man sie im Kampf gegen die Pest bereits seit dem Spätmittelalter regelmäßig einsetzte. ${ }^{10}$ Der Souverän - so die Botschaft - gewährt seinen Untertanen Schutz, verlangt im Gegenzug dafür aber Folgebereitschaft.

Im modernen Verfassungsstaat, der sich während des langen 19. Jahrhunderts im Westen herauszubilden begann, ${ }^{11}$ können die zu Bürgern gewordenen Untertanen gegenüber dem Souverän und seinen ausführenden Organen jedoch liberale Grundrechte geltend machen. Die Freiheit des Einzelnen beschränkt den staatlichen Zugriff. ${ }^{12}$ John Locke, einer der wichtigsten Vordenker des politischen Liberalismus, hatte bereits 1689 vor einer maßlosen Herrschaftsausübung des Staates gewarnt. ${ }^{13}$ Individuelle Freiheitsrechte seien durch eine Verfassung zu schützen, die sowohl die Teilung wie die Reichweite der durch den Gesellschaftsvertrag legitimierten Staatsgewalt bestimmen solle. Das Schwert, mit dem der Souverän seine weltliche Macht behauptet, hat etwas von seiner Schärfe verloren.

8 Vgl. Bredekamp, Horst: Thomas Hobbes - Der Leviathan: Das Urbild des modernen Staates und seine Gegenbilder, 1651-2001, Berlin: Akademie-Verlag 2012.

9 Darauf hat Falk, Francesca: »Hobbes' Leviathan und die aus dem Blick gefallenen Schnabelmasken«, in: Leviathan 39(2) (2011), S. 247-266 aufmerksam gemacht; Detailabbildungen finden sich dort.

10 Vgl. Dinges, Martin: »Süd-Nord-Gefälle in der Pestbekämpfung. Italien, Deutschland und England im Vergleich«, in: Wolfgang U. Eckart/Robert Jütte (Hg.), Das europäische Gesundheitssystem. Gemeinsamkeiten und Unterschiede in historischer Perspektive, Stuttgart: Steiner 1994, S. 19-51.

11 Mit Blick auf die europäische Geschichte vgl. dazu Reinhard, Wolfgang: Geschichte der Staatsgewalt. Eine vergleichende Verfassungsgeschichte Europas von den Anfängen bis zur Gegenwart, München: Beck 2000, insb. S. 410-425.

$12 \mathrm{Zu}$ der hiermit in Verbindung stehenden Herausbildung der Vorstellung von Privatheit vgl. Geuss, Raymond: Privatheit. Eine Genealogie, Frankfurt a.M.: Suhrkamp 2013.

13 Vgl. Locke, John: Zwei Abhandlungen über die Regierung, hg. v. Walter Euchner, Frankfurt a.M.: Suhrkamp 1977 [1689], insb. S. 283-311. 
Noch in einem weiteren Punkt wandelte sich im Verlauf des 19. Jahrhunderts der Charakter staatlicher Herrschaft: Nach Michel Foucault entstand im Übergang zur Moderne ein neuartiger Machttypus, der im Unterschied zur souveränen Macht, wie sie Hobbes paradigmatisch beschrieben hat, auf eine systematische Bewirtschaftung und Steigerung des Lebens abzielte: die Biomacht. »Jahrtausende hindurch ist der Mensch das geblieben, was er für Aristoteles war: ein lebendes Tier, das auch einer politischen Existenz fähig ist.« Im Gegensatz dazu sei »[d]er moderne Mensch [...] ein Tier, in dessen Politik sein Leben als Lebewesen auf dem Spiel steht «. ${ }^{14}$ Der Eintritt des natürlichen Lebens in den politischen Raum geschah auf mehreren Wegen: Neben dem Sexualitätsdispositiv, das zusammen mit dem neuzeitlichen Kriegsdiskurs bei Foucault im Mittelpunkt der Betrachtung steht, ${ }^{15}$ besaß auch der Seuchenschutz, wie ihn der Staat gemeinsam mit Medizinern, Ingenieuren, Erziehern und Statistikern seit dem 19. Jahrhundert systematisch zu betreiben begann, ${ }^{16}$ einen zentralen Stellenwert hierbei. $» D i e$ Tatsache des Lebens«, so die Folge dieser Entwicklungen, »ist nicht mehr der unzugängliche Unterbau, der nur von Zeit zu Zeit, im Zufall und in der Schicksalhaftigkeit des Todes ans Licht kommt. Sie wird zum Teil von der Kontrolle des Wissens und vom Eingriff der Macht erfaßt. « ${ }^{17}$ Die Seuchenärzte, die jetzt zusammen mit weiteren Akteuren die Gesundheit des body politic überwachen, haben in gewisser Weise an Größe gewonnen, so dass sie im Herrschaftsbild nicht mehr zu übersehen sind.

Die Idylle des modernen Staates, der seinen Bürgerinnen und Bürgern sowohl Freiheit als auch Schutz gewährt, wird jedoch durch einen Konflikt getrübt, der sich immer wieder aufs Neue in seinem Zentrum entfacht: Häufig steht der Staat nämlich vor der Situation, zwischen individuellen Freiheits- und kollektiven Schutzrechten abwägen zu müssen, da sich nicht beide zugleich garantieren lassen. Auch wenn im liberalen Verfassungsstaat das Schwert des Souveräns stumpfer geworden ist, kommt es im Zeichen biopolitischer Bevölkerungsregulation doch immer wieder zum Einsatz. »Anstelle der Drohung mit dem Mord«, beschreibt Foucault diesen Zusammenhang, »ist es nun die Verantwortung für das Leben, die der Macht Zugang zum Körper verschafft. ${ }^{18}$ Wie weit darf man

14 Foucault, Michel: Der Wille zum Wissen. Sexualität und Wahrheit 1, Frankfurt a.M.: Suhrkamp 1983, S. 138.

15 Vgl. neben ebd. auch Foucault, Michel: In Verteidigung der Gesellschaft. Vorlesungen am Collège de France (1975-1976), Frankfurt a.M.: Suhrkamp 2002.

16 Vgl. Baldwin, Peter: Contagion and the State in Europe, 1830-1930, Cambridge: Cambridge University Press 2005.

17 M. Foucault: Der Wille zum Wissen, S. 138.

18 Ebd. 
den öffentlichen Personen- und Warenverkehr einschränken, um die Ausbreitung von Infektionskrankheiten zu verhindern? Unter welchen Bedingungen lassen sich medizinische Untersuchungen, Schutzimpfungen und Heilbehandlungen, Entseuchungen und Entwesungen staatlich anordnen? Und sollen neben Berufsund Tätigkeitsverboten auch Zwangsunterbringungen auf Isolierstationen erlaubt sein, wenn sie dem allgemeinen Gesundheitsschutz dienen? Die Einschränkung individueller Freiheitsrechte mag zwar im modernen Verfassungsstaat unter einem erhöhten Rechtfertigungsdruck stehen, vollkommen unmöglich geworden ist sie aber nicht.

\section{NationalstaAtliche SOUVERÄNitÄt UND GLOBALE SEUCHENGEFAHREN}

Neben dem Konflikt zwischen individuellen Freiheits- und kollektiven Schutzrechten hat der souveräne Nationalstaat mit einem weiteren Problem zu kämpfen: Infektionskrankheiten machen nicht an Staatsgrenzen halt. Vielmehr zirkulieren Krankheitserreger, ohne auf politische und administrative Einflusssphären Rücksicht zu nehmen. Was Gilles Deleuze und Félix Guattari für Rhizome allgemein aussagen, trifft auf die Ausbreitung von Ansteckungsketten ganz besonders zu: Indem sie netzwerkartig in alle Richtungen wuchern, zeitigen sie einen >Deterritorialisierungseffekt $<{ }^{19}$ Die Geschichte des globalen Seuchenschutzes ist daher auch eine Geschichte der Grenzen souveräner Macht - und des Versuchs, dennoch handlungsfähig zu bleiben.

Nicht zuletzt unter dem Eindruck von fünf Cholerapandemien mit zum Teil verheerenden Folgen gewann im Verlauf des 19. Jahrhunderts das Thema des Seuchenschutzes an Bedeutung. ${ }^{20}$ Jede Epidemie führte den europäischen Staaten erneut vor Augen, dass sie keine voneinander abgeschotteten Gesellschaften waren, die nur gelegentlich und punktuell miteinander in Kontakt traten. Vielmehr bildeten sie einen gemeinsamen Sozialraum mit einem hohen Grad an innerer Dichte und einer zunehmenden Verflechtung nach außen. Während in den frühen 1830er Jahren Russland, Preußen und die Habsburgermonarchie die europäische Ostgrenze noch durch einen umfassenden Militärkordon von der aus Indien herannahenden Cholera abzuschirmen versuchten, nahm man im Laufe

19 Vgl. Deleuze, Gilles/Guattari, Félix: Tausend Plateaus, Berlin: Merve 1992, S. $19 \mathrm{ff}$.

20 Vgl. P. Baldwin: Contagion and the State in Europe, S. 37-243; Rosenberg, Charles E.: The Cholera Years: The United States in 1832, 1849, and 1866, Chicago: University of Chicago Press 1987. 
des 19. Jahrhunderts zusehends Abstand von Präventionsmaßnahmen dieser Art. ${ }^{21}$ Durchlässigere und gezieltere Formen der Grenzkontrolle traten an deren Stelle; die Assanierung der Städte und die Verbesserung der Individualhygiene gewannen an Bedeutung. Denn trotz des umfassenden Versuchs der Absperrung grassierte die Cholera in Europa. Die Befürworter des Sperrgürtels mussten einsehen, dass sein gesundheitlicher Nutzen gering war, wohingegen die wirtschaftlichen Kosten enorme Ausmaße annahmen. Mit dem stehenden Heer zählte zwar eine neuzeitliche Institution zu den Hauptakteuren, dennoch erwies sich die Maßnahme insgesamt als anachronistisch. Die zunehmende Verdichtung des europäischen Sozialraums bewirkte nicht nur die sprunghafte Zunahme von Epidemien, sondern bildete auch ein massives Hindernis bei der Abschottung ganzer Regionen.

Auch wenn Krankheitserreger nicht an den Grenzen von Nationalstaaten haltmachen, endet an ihnen aber das Herrschaftsgebiet eines Souveräns und das schwierige Terrain der zwischenstaatlichen Diplomatie beginnt. Mit den Internationalen Sanitätskonferenzen, die in den Jahren zwischen 1851 und 1938 vierzehn Mal stattfanden, wurden erste Versuche unternommen, auf diplomatischem Wege ein globales Seuchenregime aufzubauen. ${ }^{22}$ Standen die ersten Treffen noch ganz unter dem Eindruck der Cholera, lässt sich seit den 1880er Jahren eine thematische Öffnung erkennen. Vereinbarungen im Bereich von Quarantäne-, Inspektions- und Überwachungsmaßnahmen konnten mühsam erarbeitet und vereinzelt ratifiziert werden. Ein weiterer Schritt in dieser Richtung war die Gründung von Tropeninstituten, welche die Übergangszonen zwischen dem kolonialisierenden Zentrum und der kolonialisierten Peripherie regulieren sollten. Neben den 1899 in London und Liverpool eröffneten Forschungseinrichtungen ist das 1900 eingerichtete Hamburger Institut für Schiffs- und Tropenkrankheiten hervorzuheben. ${ }^{23}$ In einer Kombination aus Grundlagenforschung und Ärzteausbildung, Krankenbehandlung und Seuchenprophylaxe, Laienaufklärung und

21 Vgl. nochmals P. Baldwin: Contagion and the State in Europe, S. 37-243 sowie Briese, Olaf: Angst in den Zeiten der Cholera. Über kulturelle Ursprünge des Bakteriums. Seuchen-Cordon I, Berlin: Akademie-Verlag 2003, S. 233-280.

22 Vgl. Howard-Jones, Norman: The Scientific Background of the International Sanitary Conferences 1851-1938, Genua: WHO 1975; Bynum, William F.: »Policing Hearts of Darkness: Aspects of the International Sanitary Conferences«, in: History and Philosophy of the Life Sciences 15(3) (1993), S. 421-434.

23 Vgl. dazu Wulf, Stefan: Das Hamburger Tropeninstitut 1919 bis 1945. Auswärtige Kulturpolitik und Kolonialrevisionismus nach Versailles, Berlin/Hamburg: Reimer 1994. 
Politikberatung suchte man den gefürchteten Ansteckungskrankheiten entgegenzutreten.

Jedoch erhielt die globale Gesundheitspolitik erst mit der Weltgesundheitsorganisation, die 1948 als Sonderbehörde der Vereinten Nationen eingerichtet wurde, eine Koordinations- und Steuerungseinheit. ${ }^{24}$ Die souveränen Nationalstaaten nutzten den im Kleinen bereits vielfach bewährten Mechanismus der Organisationsbildung, ${ }^{25}$ um ihre Zusammenarbeit auf diesem Gebiet abzustimmen. Während Hobbes zufolge »Bündnisse von Untertanen« innerhalb eines Staates »einen Beigeschmack von ungesetzlicher Absicht« besitzen, sofern sie in staatliche Aufgaben- und Hoheitsbereiche hineinzuwirken versuchen, »sind Bündnisse zwischen Gemeinwesen, über denen keine menschliche Macht errichtet ist, um sie alle in Schrecken zu halten, nicht nur gesetzlich, sondern auch nützlich, solange sie dauern $\ll .{ }^{26}$ Mit der Weltgesundheitsorganisation (und anderen Einrichtungen dieser Art) wurde, wenn man so möchte, ein künstlicher Körper zweiter Ordnung erschaffen, mit dem die Mitgliedsstaaten, die ihn als künstlichen Körper erster Ordnung trugen, eine globale Handlungsfähigkeit im Bereich der internationalen Gesundheitspolitik zu erlangen versuchten. ${ }^{27}$ Den souveränen Nationalstaaten war es nunmehr im Prinzip möglich, auf Ebene der sich konsolidierenden Weltgesellschaft kollektiv verbindliche Entscheidungen zu treffen.

Mit den 1969 erlassenen Internationalen Gesundheitsvorschriften, welche die Internationalen Sanitätsvorschriften aus dem Jahr 1951 ablösten, etablierte die Weltgesundheitsorganisation völkerrechtlich verbindliche Standards und Normen auf diesem Gebiet. ${ }^{28}$ Das ausdrückliche Ziel der Regularien bestand darin, sthe maximum security against the international spread of disease with the mi-

$24 \mathrm{Zu}$ den unmittelbar vorausgegangenen Entwicklungen vgl. Bashford, Alison: »Global Biopolitics and the History of World Health«, in: History of the Human Sciences 19(1) (2006), S. 67-88.

25 Bei Organisationen handelt es sich um einen bestimmten Typus von Sozialsystem, für den formale Mitgliedschaftsverhältnisse und an diese gebundene Entscheidungen konstitutiv sind; vgl. grundlegend dazu Luhmann, Niklas: Funktionen und Folgen formaler Organisation, Berlin 1964. Zumeist wird hierbei stillschweigend unterstellt, dass lediglich natürliche Personen Organisationsmitglieder sind. Wie jedoch interund supranationale Organisationen zeigen, können auch - im weitesten Sinne - juristische Personen Mitglied einer formalen Organisation sein.

26 Alle Zitate in T. Hobbes: Leviathan, S. 200.

$27 \mathrm{Zu}$ solchen und anderen >lateralen Weltsystemen dien zur atopischen Gesellschaft, Frankfurt a.M.: Suhrkamp 2001, S. 107-191.

28 Vgl. WHO: »International Sanitary Regulations, 25. Mai 1951«, in: Treaty Series 22 (1962); WHO: International Health Regulations, 25. Juli 1969, Genf: WHO 1983. 
nimum interference with world traffic $\ll^{29}$ zu gewährleisten. Zu diesem Zweck habe man auf Weltebene ein epidemiologisches Überwachungs- und Frühwarnsystem zu installieren; im Einzelnen sei mithilfe von Telefax, Telegramm und Telefon eine zügige Nachrichtenübertragung zu gewährleisten. War die Verbreitung der Kommunikation schneller als die Verbreitung der Erreger, entstand eine zeitliche Differenz, die sich präventiv nutzen ließ. Neben der Einrichtung eines globalen Kommunikationsnetzes sollten Gesundheitskontrollen in Form von Selbstauskünften, ärztlichen Untersuchungen und Impfpässen helfen, die Träger von Krankheitserregern ausfindig zu machen. Eine besondere Aufmerksamkeit erfuhren hierbei See- und Flughäfen, die als Knotenpunkte der globalen Zirkulation in strategischer Hinsicht von zentraler Bedeutung waren.

Daneben unternahm die Weltgesundheitsorganisation Anstrengungen, einzelne Infektionskrankheiten gezielt zu bekämpfen. Eines der ersten großen Projekte in diesem Bereich war das Global Malaria Eradication Program. Um die gefährliche Tropenkrankheit, die von der Anopheles-Mücke übertragen wird, zu besiegen, setzte man zwischen 1955 und 1969 in rund 60 Ländern der Welt wiederholt und flächendeckend das Insektizid DDT ein. Jedoch musste man schon bald erkennen, dass das Vorhaben, die Krankheit auszurotten, trotz großer Erfolge in einzelnen Regionen, deutlich zu ambitioniert gewesen war. ${ }^{30}$ Anders verhielt es sich bei den Pocken, denen die Weltgesundheitsorganisation 1959 den Kampf ansagte. ${ }^{31}$ Das bis heute umfassendste Impfprogramm in der Menschheitsgeschichte hat nach rund 20 Jahren sein Ziel erreicht. 1980 konnte die Weltgesundheitsorganisation verkünden: »smallpox is dead! « ${ }^{32}$ Wie im entsprechenden Beschluss der Weltgesundheitsversammlung festgehalten wurde, haben die verschiedenen Nationen durch eine gemeinsame Anstrengung die Menschheit von einen ihrer größten Geißeln befreit. ${ }^{33}$

Nur zwei Jahre, nachdem die Menschheit bei den Pocken bewiesen hatte, dass sich der Kampf gegen Infektionskrankheiten mit vereinten Kräften gewin-

29 WHO: »International Sanitary Regulations and Additional Amending Regulations«, S. 5 .

30 Vgl. Zimmer, Thomas: »In the Name of World Health and Development: The World Health Organization and Malaria Eradication in India, 1949-1970«, in: Marc Frey/ Sönke Kunkel/Corinna Unger (Hg.), International Organizations and Development, 1945-1990, London: Palgrave Macmillan 2014, S. 126-149.

31 Vgl. dazu die umfassende Darstellung in Fenner, Frank et al.: Smallpox and its Eradication, Genf: WHO 1988, insb. S. 365-592, 1103-1148.

32 So das jubilatorische Titelcover des WHO-Magazins vom Mai 1980; eine Abbildung findet sich ebd., S. 1106.

33 Vgl. ebd., S. 1135. 
nen ließ, erschien eine neue Seuchengefahr auf dem epidemiologischen Radar: Das von der US-amerikanischen Seuchenbehörde einberufene Gesundheitsforum in Washington verkündete am 27. Juli 1982, dass man fortan jene mysteriöse Viruserkrankung, die seit rund einem Jahr in den Metropolen des Landes vermehrt aufgetreten war, als `Acquired Immunodeficiency Syndrome`, kurz AIDS, bezeichnen werde. ${ }^{34}$ Spätestens zu diesem Zeitpunkt bildete die Krankheit einen öffentlichen, sozialen Tatbestand. Die bis dahin vorherrschende Erzählung von einem nahenden Ende des Seuchenzeitalters entpuppte sich als gefährliche Selbsttäuschung. AIDS schockierte, weil mit großer Plötzlichkeit grundlegende Annahmen über den eigenen Ort in der Geschichte verabschiedet werden mussten. »Seuchen wie Pest und Cholera, folgenschwere Viruserkrankungen wie Pocken und Kinderlähmung«, hieß es beispielsweise 1983 im Spiegel, »hat der Mensch durch zunehmende Hygiene, durch Impfstoffe und Medikamente unter Kontrolle gebracht. Auch über Viruskrankheiten wie Herpes und Hepatitis rückt der Sieg schon in greifbare Nähe - wie ein Schock kommt da die geheimnisvolle Krankheit Aids. $\ll^{35}$

Wie die Vereinten Nationen in ihrer Millenniumserklärung mit Nachdruck betont haben, sei es angesichts der globalen Herausforderungen der Gegenwart die Pflicht aller Länder, durch eine gemeinsame Anstrengung eine friedlichere und gerechtere Welt zu schaffen. ${ }^{36}$ Der Kampf gegen HIV/AIDS, Malaria und weitere Krankheiten bildete hierbei eines von acht Entwicklungszielen, die bis zum Jahr 2015 erreicht werden sollten. ${ }^{37}$ Laut UNAIDS starben im Jahr 2000 weltweit 1,6 Millionen Menschen an den Folgen der Immunschwächekrankheit, wobei rund $80 \%$ der Todesfälle im subsaharischen Afrika zu verzeichnen waren. ${ }^{38}$ Bis 2004 stieg die Zahl der jährlichen AIDS-Toten auf 2 Millionen an, bevor sie langsam, aber stetig zurückging. Im Jahr 2015 sind weltweit 1,2 Millio-

34 Zur frühen Phase der Epidemie vgl. Grmek, Mirko D.: A History of AIDS: Emergence and Origin of a Modern Pandemic, Princeton: Princeton University Press 1990, S. 3-43; Epstein, Steven: Impure Science: AIDS, Activism, and the Politics of Knowledge, Berkeley: University of California Press 1996, S. 45-78.

35 Anonymus: »Aids: ১Eine Epidemie, die erst beginnt««, in: Der Spiegel 38(23) (1983), S. 144-163, hier S. 147.

36 Vgl. UN-Generalversammlung: United Nations Millennium Declaration, New York: UN 2000.

37 Vgl. http://www.un.org/millenniumgoals/ [letzter Aufruf am 31. Januar 2017].

38 Die Angaben stammen aus der Datenbank auf http://aidsinfo.unaids.org/ [letzter Aufruf am 31. Januar 2017]. Die im Ereigniszeitraum selbst verbreiteten Zahlen lagen jedoch deutlich höher; vgl. etwa UNAIDS: Report on the Global HIV/AIDS Epidemic, Genf: UNAIDS 2000, S. 124. 
nen Menschen an der Krankheit gestorben, was man aufgrund der rückläufigen Entwicklung als großen Erfolg wertete. ${ }^{39}$ Jedoch hatte AIDS zu diesem Zeitpunkt bereits 35 Millionen Menschenleben gefordert.

\section{ZUKUNFTSBEZUG UND ORGANISATIONSFORM DES NEUEN SEUCHENREGIMES}

Im Schatten der globalen AIDS-Krise, die durch humanitäre Maßnahmen in den Griff zu bekommen versucht wurde und wird, hat sich nach der Jahrtausendwende auf Weltebene ein neuartiges Seuchenregime herauszubilden begonnen, das auf hochansteckende Infektionskrankheiten gerichtet ist. ${ }^{40}$ Diese gilt es nicht mehr auszurotten, wohl aber einzudämmen. Das zentrale Anliegen besteht darin, sich auf Pandemien vorzubereiten, deren Auftreten man unter den Bedingungen globaler Vergesellschaftung als letztlich unvermeidbar erachtet. Jedoch führt diese Erwartungshaltung keineswegs zu einem abwartenden Verhalten, sondern wird im Gegenteil von einer erhöhten Alarm- und Aktionsbereitschaft begleitet. Das neue Seuchenregime verfolgt somit eine Doppelstrategie: Durch ein frühzeitiges Eingreifen versucht man, dem möglichen Schaden zuvorzukommen, macht sich aber zugleich auf ein Versagen der primären Schutzmaßnahmen gefasst.

Den Grundstein für das neue Seuchenregime hat die Weltgesundheitsorganisation mit dem im Jahr 2000 eingerichteten Global Outbreak Alert and Response Network (GOARN) gelegt, das seither in mehr als 120 Fällen aktiv geworden ist. ${ }^{41}$ Durch $»$ rapid identification, verification and communication of threats leading to coordinated response ${ }^{42}$ soll der internationalen Ausbreitung von Infektionskrankheiten zeitnah entgegengewirkt werden. »No single institution or country«, so das Hauptargument für die länderübergreifende Vernetzung, »has all of the capacities to respond to international public health emergencies caused

39 Vgl. WHO/UNAIDS: Global AIDS Response Progress Reporting 2015, Genf: UNAIDS 2015, S. 4.

40 Vgl. Lakoff, Andrew: »Two Regimes of Global Health«, in: Humanity 1(1) (2010), S. 59-79.

41 Vgl. http://www.who.int/csr/disease/ebola/partners/en/ [letzter Aufruf am 31. Januar 2017].

42 WHO: Global Outbreak Alert and Response: Report of a WHO Meeting, Genf 2000, S. 2. 
by epidemics and by new and emerging infectious diseases. $\aleph^{43}$ Dass die SARSPandemie von 2002/03, bei der knapp 800 Todesfälle registriert wurden, einen globalen Alarm auslöste, da man die Gesundheit der Weltbevölkerung bedroht sah, ging nicht zuletzt auf das GOARN zurück. Die Risikokommunikation, die sich am Ansteckungspotenzial entfachte, hat in diesem Fall die Ansteckungswirklichkeit überholt. ${ }^{44}$

Auch die 2005 grunderneuerten Internationalen Gesundheitsvorschriften, die im Juni 2007 in Kraft traten, haben die institutionell verankerte Alarm- und Aktionsbereitschaft deutlich erhöht. Im Unterschied zu den Vorläuferregularien von 1951 und 1969, die noch vergleichsweise statisch auf einen festen Katalog von Infektionskrankheiten bezogen waren, soll nunmehr jeder potenzielle >Public Health Emergency of International Concern (PHEIC) mithilfe eines sensiblen Frühwarnsystems aufgespürt werden, wobei ein besonderes Augenmerk auf den Durchgangspunkten (`points of entry $\measuredangle$ ) der globalen Zirkulation liegt. ${ }^{45}$ Die Mitgliedsstaaten der Weltgesundheitsorganisation tragen die Verantwortung für die lokale Implementierung des globalen Sicherheitsnetzes; im Einzelnen haben sie Überwachungs-, Kontakt- und Koordinationsstellen einzurichten. ${ }^{46}$ In Deutschland ist seit 2010 das Gemeinsame Melde- und Lagezentrum von Bund und Ländern, das dem Bundesamt für Bevölkerungsschutz und Katastrophenhilfe untersteht, zusammen mit dem Robert-Koch-Institut federführend auf diesem Gebiet tätig. ${ }^{47}$ Um den potenziellen Gesundheitsschaden, der von einem regionalen

43 WHO: "Global Outbreak Alert and Response Network-GOARN, Partnership in Outbreak Response«, abrufbar unter: http://www.who.int/csr/outbreaknetwork/goa rnenglish.pdf [letzter Aufruf am 31. Januar 2017].

44 Vgl. dazu Ong, Aihwa: »Assembling Around SARS: Technology, Body Heat, and Political Fever in Risk Society«, in: Angelika Poferl/Nathan Snaider (Hg.), Ulrich Becks kosmopolitisches Projekt. Auf dem Weg in eine andere Soziologie, Baden-Baden: Nomos 2004, S. 81-89; Pohler, Wiebke: »SARS - Ein globales Risikoereignis«, in: Wiebke Pohler/Markus Holzinger/Stefan May (Hg.), Weltrisikogesellschaft als Ausnahmezustand, Weilerswist: Velbrück Wissenschaft 2010, S. 121-151.

45 Vgl. WHO: International Health Regulations, Genf: WHO 2005, S. 11-15, 18-20. Zur Novellierung der Internationalen Gesundheitsvorschriften vgl. Davies, Sara E./ Kamradt-Scott, Adam/Rushton, Simon: Disease Diplomacy: International Norms and Global Health Security, Baltimore 2015; Gostin, Lawrence O.: Global Health Law, Cambridge, Mass: Harvard University Press 2014, S. 175-204.

46 Vgl. WHO: International Health Regulations, S. 11.

$47 \mathrm{Zu}$ den rechtlichen Grundlagen vgl. „Gesetz zu den Internationalen Gesundheitsvorschriften (2005) (IGV) vom 23. Mai 2005. Vom 20. Juli 2007«, in: Bundesgesetzblatt 103 (2007), S. 930-994; »Gesetz zur Durchführung der Internationalen Gesundheits- 
Krankheitsgeschehen für die Weltbevölkerung ausgeht, richtig einschätzen zu können, hat die Weltgesundheitsorganisation verbindliche Richtlinien erlassen. ${ }^{48}$ Die Risikobeurteilung vor Ort soll weltweit einheitlichen Standards folgen, nur so könne die globale Anschlussfähigkeit der lokal generierten Informationen und Entscheidungen gewährleistet werden. Dies verhindert freilich nicht, dass es zumindest in der Rückschau betrachtet zu Fehleinschätzungen kommen kann. So hat die Weltgesundheitsorganisation die Ebolavirus-Epidemie von 2014/15 erst vergleichsweise spät als einen PHEIC eingestuft. ${ }^{49}$

Zusammen mit den Aufmerksamkeitsschwellen wird versucht, die Reaktionszeiten herabzusenken. Bildet die dezentrale Struktur von Netzwerken hinsichtlich der Alarmfunktion einen Vorteil, insofern sie die Aufmerksamkeit im Raum verteilt, wirkt sich das fehlende Handlungszentrum für die Interventionsfunktion hingegen nachteilig aus. Aller Netzwerkrhetorik zum Trotz wird daher keineswegs auf die altbewährten Strukturprinzipien von Hierarchie und Arbeitsteilung verzichtet. Die Internationalen Gesundheitsvorschriften etwa sehen vor, dass die zuständigen Einheiten vor Ort bei einer positiven Risikoeinschätzung binnen 24 Stunden die Weltgesundheitsorganisation benachrichtigen, welche dann in einem hierarchisch und arbeitsteilig organisierten Prozess die benötigten Informationen und Experten in die betroffenen Regionen zu versenden hat. ${ }^{50} \mathrm{An}$ jeder Stelle im Gesamtprozess wird flexibel auf die Strukturprinzipien zurückgegriffen, die das beste Teilergebnis versprechen. Durch eine Kombination von Netzwerk, Hierarchie und Arbeitsteilung soll ein zügiges Eingreifen ermöglicht werden.

Trotz der großen Anstrengungen, die man auf Weltebene unternimmt, um Pandemien zu verhindern, wird ihr Eintreffen als weitestgehend sicher erachtet. Der nächste Gesundheitsnotstand komme bestimmt. Schon 2005 gab die Weltgesundheitsorganisation zu bedenken: »Influenza experts agree that another pandemic is likely to happen but are unable to say when. The specific characteristics of a future pandemic virus cannot be predicted. Nobody knows how pathogenic a

vorschriften (2005) und zur Änderung weiterer Gesetze. Vom 21. März 2013«, in: Bundesgesetzblatt 109 (2013), S. 566-584.

48 Die maßgebliche Entscheidungsmatrix findet sich in ebd., S. 43-46. Für weitergehende Bestimmungen vgl. WHO: Rapid Risk Assessment of Acute Public Health Events, Genf: WHO 2012.

49 Für eine ausführliche Betrachtung dieses Falls vgl. die Beiträge in Andrew Lakoff/Stephen J. Collier/Christopher Kelty (Hg.), Ebola's Ecologies (limn 5) (2015).

$50 \mathrm{Zu}$ den genauen Bestimmungen vgl. WHO: International Health Regulations, S. 11$15,31-34,40-42$. 
new virus would be, and which age groups it would effect. $\ll^{51}$ Die Unvorhersagbarkeit ist demnach keine absolute sondern eine relative, so dass man sich zumindest im Großen und Ganzen auf das Unvermeidliche - die nächste Pandemie - einstellen kann. ${ }^{52}$ In einer Hochglanzbroschüre zum Thema präsentierte 2006 auch das United States Department of Homeland Security eine ähnliche Sicht der Dinge: »Some will say«, so der zitierte US-amerikanische Gesundheitsminister Mike Leavitt, »this discussion of the Avian Flu is an overreaction. Some may say, >Did we cry the wolf? ใ The reality is that if the H5N1 virus does not trigger pandemic flu, there will be another virus that will. $\varkappa^{53}$ Auch wenn niemand vorherzusagen vermöge, wann und wo die nächste Pandemie zuschlägt, könne zumindest als sicher gelten, dass sie kommt.

Damit folgt auch der globale Seuchenschutz einem breiten Trend, den Notbeziehungsweise Unfall zu normalisieren. Bereits in den 1970er Jahren und nochmals verstärkt nach der Jahrtausendwende imaginiert man in verschiedenen Bereichen des öffentlichen Lebens, die Zukunft als tendenziell katastrophisch. ${ }^{54}$ Obwohl sich der gesellschaftliche Erwartungshorizont unter dem Eindruck ökologischer, politischer und wirtschaftlicher Krisen zunehmend verfinstert, lässt man jedoch nicht alle Hoffnung auf Prävention fahren. Zwar sei das Auftreten von Pandemien letzten Endes unvermeidbar, dennoch könne versucht werden, das Schadensausmaß möglichst gering zu halten. Es gilt, mit anderen Worten, für den Notfall zu planen, um ihm ein Stück weit seine Ereignisschwere zu nehmen. Genau dies meint das Konzept der preparedness: ein Handeln, Entscheiden und Regieren unter den Bedingungen von Unsicherheit. ${ }^{55}$ "Although it is not considered feasible to halt the spread of a pandemic virus«, gab 2005 die Welt-

51 WHO: WHO Checklist for Influenza Pandemic Preparedness Planning, Genf: WHO 2005, S. VIf.

52 Vgl. dazu aus ethnographischer Perspektive auch MacPhil, Theresa: »A Predictable Unpredictability: The 2009 H1N1 Pandemic and the Concept of >Strategic Uncertainty< within Global Public Health«, in: Behemoth 3(3) (2010), S. 57-77.

53 Mike Leavitt zit. n. Department of Homeland Security: Pandemic Influenza: Preparedness, Response, and Recovery. Guide for Critical Infrastructure and Key Resources, Washington: US Department of Homeland Security 2006, S. 10.

54 Vgl. neben der in der Einleitung dieses Textes aufgeführten Literatur auch Lakoff, Andrew: »Preparing for the Next Emergency«, in: Public Culture 19(2) (2007) S. 247-271.

55 Vgl. Lentzos, Filippa/Rose, Nikolas: „Governing Insecurity: Contingency Planning, Protection, Resilience«, in: Economy and Society 38(2) (2009), S. 230-254; Anderson, Ben: »Preemption, Precaution, Preparedness: Anticipatory Action and Future Geographies«, in: Progress in Human Geography 34(6), 2010, S. 777-798. 
gesundheitsorganisation zu bedenken, »it should be possible to minimize its consequences through advance preparation to meet the challenge. $«{ }^{56}$ Eine intelligente Katastrophenplanung könne somit verhindern, dass sich eine hochansteckende Krankheit im Ernstfall weiter ausbreite und kritische Infrastrukturen, deren Ausfall die nationale Sicherheit gefährdeten, zusammenbrechen. ${ }^{57}$ Lokale Public Health Emergency Operations Centres (EOCs), die 2012 von der Weltgesundheitsorganisation zu einem globalen Netzwerk (EOC-Net) zusammengeschlossen wurden, um Synergie- und Isomorphieeffekte zu erzielen, tragen den Planungsprozess. ${ }^{58}$ Neben Bestandsaufnahmen, welche die zur Verfügung stehenden Ressourcen und Kontingente erfassen, besitzen Szenario-Techniken, ${ }^{59}$ die zunächst unabhängig von Wahrscheinlichkeitsaussagen mögliche Notfallsituationen imaginieren, um sich auf diese einstellen zu können, eine zentrale Bedeutung hierbei.

Das Konzept der preparedness arbeitet mit zwei Klassen von Schadensfällen: Erstschäden, die sich nicht verhindern lassen, und vermeidbaren Folgeschäden. Insofern handelt es sich um ein Zurückweichen des präventiven Horizonts, nicht aber um dessen Zusammenbruch oder Verabschiedung. Auf dem Weg der Katastrophenplanung könne man die Widerstandskraft der Weltgemeinschaft stärken und so den »social, economic, and political stress «, ${ }^{60}$ der aus Pandemien resultiere, möglichst gering halten. Der UN-Generalsekretär Ban Ki-moon hat in diesem Zusammenhang auf der 62. Weltgesundheitsversammlung im Mai 2009 die Frage aufgeworfen: »how do we build resilience in an age of unpredictability

56 WHO: WHO Global Influenza Preparedness Plan: The Role of WHO and Recommendations for National Measures Before and During Pandemics, Genf: WHO 2005, S. 3 .

$57 \mathrm{Zu}$ dem hiermit zusammenhängenden Konzept der biosecurity, das den Gesundheitsmit dem Sicherheitsdiskurs verzahnt, vgl. Fidler, David P./Gostin, Lawrence O.: Biosecurity in the Global Age: Biological Weapons, Public Health, and the Rule of Law, Stanford: Stanford Law and Politics 2008; Andrew Lakoff/Stephen J. Collier (Hg.), Biosecurity Interventions: Global Health \& Security in Question, New York: Columbia University Press 2008.

58 Vgl. WHO: Framework for a Public Health Emergency Operations Centre, Genf: WHO 2015.

59 Vgl. zu diesen auch Steinmüller, Angela/Steinmüller, Karlheinz: Ungezähmte Zukunft. Wild Cards und die Grenzen der Berechenbarkeit, München: Gerling Akademie Verlag 2003.

60 So die Weltgesundheitsorganisation auf ihrer Homepage zum Konzept der pandemic preparedness, abrufbar unter: http://www.who.int/influenza/preparedness/pandemic/ en/ [letzter Aufruf am 31. Januar 2017]. 
and interconnection? « Durch Notfallplanung, lautete seine Antwort. »This is how we will make the global community more resilient. This is how we ensure that wherever the next threat to health, peace or economic stability may emerge, we will be ready. ${ }^{61}$ Alle für das gesellschaftliche Leben zentralen Institutionen sollten sich durch eine weitsichtige Planung auf die nächste Pandemie vorbereiten, um so die allgemeine Belastbarkeit zu steigern. ${ }^{62}$ "Wir brauchen flexible Systeme«, forderte 2011 auch Nathan Wolfe, Gründer der Initiative Global Viral Forecasting, »die nicht darauf setzen, dass die nächste Bedrohung von Influenza oder SARS ausgeht oder welcher gerade angesagten Infektionskrankheit auch immer. Die Systeme sollten allgemein und auf die Zukunft ausgerichtet sein. ${ }^{63}$ Denn nur Vorhersage-, Frühwarn-, Überwachungs- und Interventionssysteme, die flexibel auf die sich fortwährend ändernden Umweltbedingungen reagieren, seien robuste Systeme. Da sich niemals alle Viren ausrotten ließen, müsse daran gearbeitet werden, zumindest ihre Verbreitungsdynamik zu kontrollieren. Durch Ausbau des globalen Seuchenschutzes habe man der immer enger zusammenrückenden Weltbevölkerung »ein globales Immunsystem $\aleph^{64}$ zu geben, welches die lokal auftretenden Infektionsherde eindämme.

Auch wenn nach Ansicht der Weltgesundheitsorganisation der globale Personen- und Güterverkehr grundsätzlich ungehindert fließen soll, müsse die Zirkulation gelegentlich und punktuell unterbrochen werden, um die Mitreise ungebetener Gäste zu verhindern. ${ }^{65}$ Tätigkeitsverbote, Reisebeschränkungen, Quarantäne- und Isolationsmaßnahmen sind daher in den entsprechenden Regularien weiterhin vorgesehen ${ }^{66}$ Zugleich werden diese Eingriffe aber als problematisch

61 Ban Ki-moon, »Resilience and Solidarity: Our Best Response to Crisis (Address to the 62nd World Health Assembly 19 May 2009)«, abrufbar unter: http://www. who.int/mediacentre/events/2009/wha62/secretary_general_speech_20090519/en/ [letzter Aufruf am 31. Januar 2017].

62 Vgl. WHO: Whole-of-Society Pandemic Readiness: WHO Guidelines for Pandemic Preparedness and Response in the Non-Health Sector, Genf: WHO 2009.

63 Wolfe, Nathan: Virus. Die Wiederkehr der Seuchen, Reinbek bei Hamburg: Rowohlt 2012 [2011], S. $276 f$.

64 Ebd., S. 290.

65 Vgl. Stephenson, Niamh: »Emerging Infectious Diseases/Emerging Forms of Biological Sovereignty«, in: Science, Technology, \& Human Values 36(5), S. 616-637; Opitz, Sven: »Regulating Epidemic Space: The nomos of Global Circulation«, in: Journal of International Relations and Development 18(1) (2015), S. 1-22.

66 Dies zeigt sich nicht nur bei den globalen Bestimmungen, sondern auch in der nationalen Gesetzgebung; vgl. nur das »Gesetz zur Neuordnung seuchenrechtlicher Vor- 
erachtet, da sie grundlegende Freiheitsrechte der Person antasten. So lautet das erste Prinzip der Internationalen Gesundheitsvorschriften: »The implementation of these Regulations shall be with full respect for the dignity, human rights and fundamental freedoms of persons. $\ll^{67}$ Auch in weiteren Bestimmungen und Dokumenten lässt sich ein hohes Maß an Problembewusstsein erkennen:

In emergency situations, the enjoyment of individual human rights and civil liberties may have to be limited in the public interest. However, efforts to protect individual rights should be part of any policy. Measures that limit individual rights and civil liberties must be necessary, reasonable, proportional, equitable, non-discriminatory and in full compliance with national and international laws. ${ }^{68}$

Auch wenn man nicht gänzlich auf seuchenpolizeiliche Maßnahmen verzichten will, lastet nunmehr ein erhöhter Rechtfertigungsdruck auf ihnen.

Damit sind einige der Elemente benannt, die für das gegenwärtig im Aufbau begriffene Seuchenregime im Bereich der globalen Pandemieplanung maßgeblich sind. Auch wenn sich bislang lediglich die ungefähren Umrisse abzeichnen, kann man doch etwas von den Ausmaßen dieses neuen Makroakteurs ${ }^{69}$ erahnen, der da langsam an der Oberfläche der Weltgesellschaft auftaucht. Hierbei hat man es jedoch mit einem Akteur zu tun, der um die Begrenztheit der eigenen Handlungsmacht weiß und aus der Erwartung, das primäre Handlungsziel Schadensfreiheit - regelmäßig nicht erreichen zu können, neue Handlungsenergie schöpft, um zumindest den worst case abzuwenden. Auch wenn sich Pandemien nicht sicher verhindern ließen, bleibe die Möglichkeit, das Schadensausmaß zu begrenzen. An die Stelle des Versprechens vollkommener Prävention tritt ein Programm zur Verwaltung von Unsicherheit. ${ }^{70}$

schriften (Seuchenrechtsneuordnungsgesetz - SeuchRNeuG). Vom 20. Juli 2000«, in: Bundesgesetzblatt 96 (2000), S. 1045-1077.

67 WHO: The International Health Regulations, S. 10.

68 WHO: Pandemic Influenza Risk Management: WHO Interim Guide, Genf: WHO 2013, S. 47.

69 Im Sinne von Callon, Michel/Latour, Bruno: »Die Demontage des großen Leviathans: Wie Akteure die Makrostruktur der Realität bestimmen und Soziologen ihnen dabei helfen«, in: Andréa Belliger/David J. Krieger (Hg.), ANThology. Ein einführendes Handbuch zur Akteur-Netzwerk-Theorie, Bielefeld: transcript 2006, S. 75-101.

$70 \mathrm{Zu}$ dieser Verschiebung im zeitgenössischen Regieren vgl. Bröckling, Ulrich: »Resilienz: Belastbar, flexibel, widerstandsfähig«, in: ders., Gute Hirten führen sanft. Über Menschenregierungskünste, Berlin: Suhrkamp 2017, S. 113-139. 
